Supporting Information. Deconvolution of amide I band of recombinant silk-silica fusion proteins (nh-15mer, 15mer-ch, nh-15mer-R5, R5-15mer-ch, nh-15mer-silaffin), FTIR spectra of nh-15mer-R5 films on different substrates.

\title{
Integrated modeling and experimental approaches to control silica modification of design silk-based biomaterials
}

Dinjaski Nina ${ }^{1,2^{*}}$, Ebrahimi Davoud ${ }^{2 *}$, Ling Shengjie ${ }^{1,2^{*}}$, Shah Suraj ${ }^{1}$, Buehler Markus J. ${ }^{2} \uparrow$, Kaplan David L. ${ }^{1} \dagger$

${ }^{1}$ Department of Biomedical Engineering, Tufts University, Medford, MA, 02155, USA

${ }^{2}$ Department of Civil and Environmental Engineering, Massachusetts Institute of Technology, Cambridge, MA, 02139, USA

$\dagger$ To whom correspondence should be addressed:

David L. Kaplan. E-mail David.Kaplan@Tufts.edu; Tel. +1 617626 3251; Fax + 6176273231

Markus J. Buehler. E-mail mbuehler@,MIT.EDU; Tel. +1 6174522750

* These authors contributed equally to this work.

Number of pages: 3; Number of figures: 2 

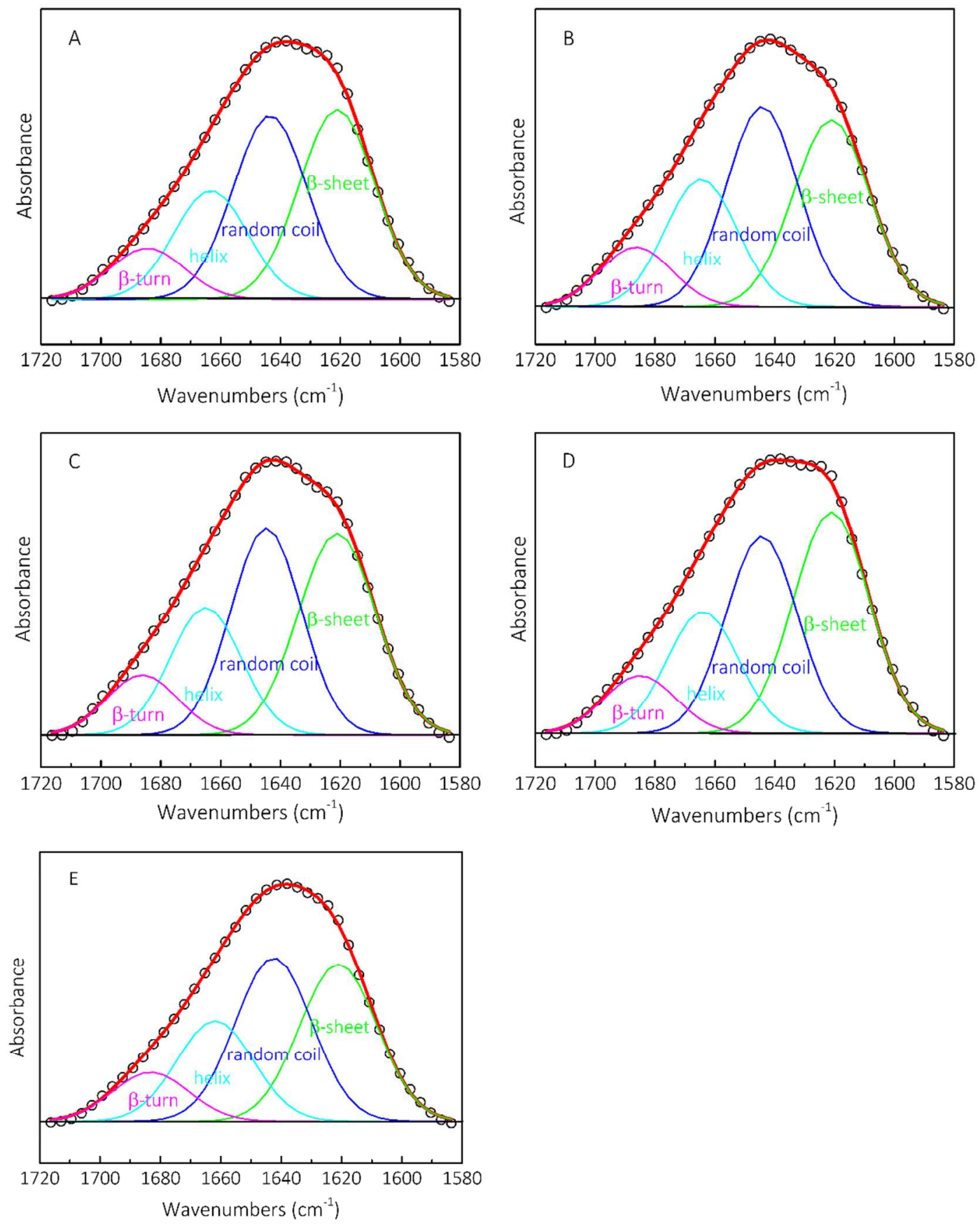

Figure S1. Typical deconvolution results of amide I band of recombinant silk-silica fusion proteins: (A) nh-15mer; (B) 15mer-ch; (C) nh-15mer-R5; (D) R5-15mer-ch; and (D) nh-15mersilaffin. All of the films were prepared in water and $\beta$-sheet formation induced via water annealing at room temperature for $24 \mathrm{~h}$. 


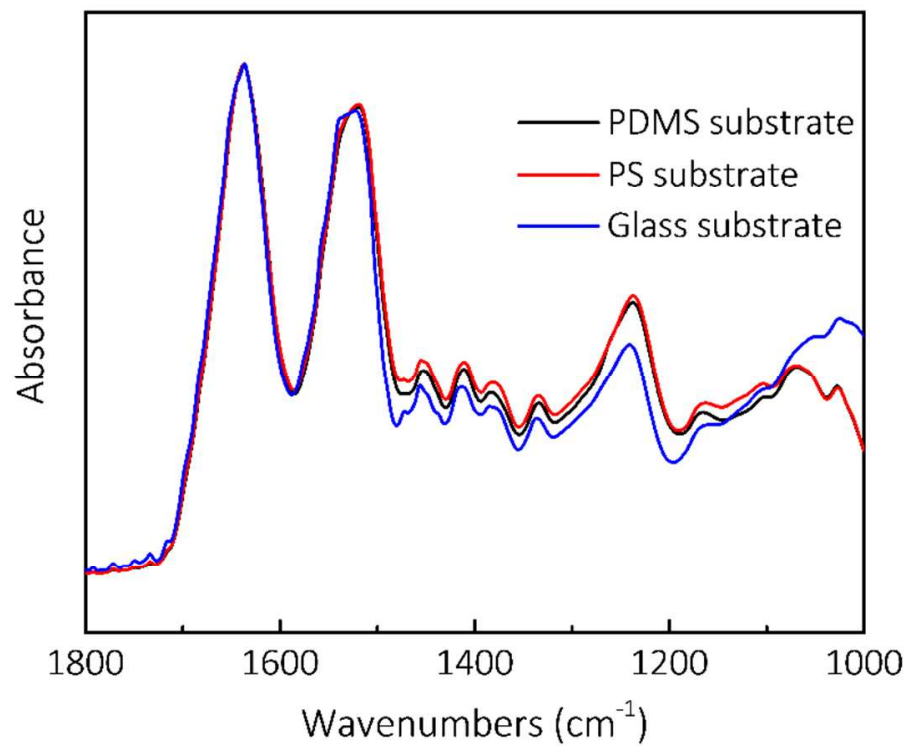

Figure S2. Normalized FTIR spectra of nh-15mer-R5 films made on polydimethylsiloxane (PDMS), polystyrene (PS) and glass substrates. 